\title{
Coffea arabica Extract
}

National Cancer Institute

\section{Source}

National Cancer Institute. Coffea arabica Extract. NCI Thesaurus. Code C154289.

An orally bioavailable dietary supplement composed of an extract from the beans of green coffee (Coffea arabica), which are unprocessed and unroasted, with potential antiinflammatory, antioxidant and chemopreventive activities. Coffea arabica extract contain high amounts of phenolic compounds and their derivatives, such as chlorogenic acid (CGA), and alkaloids, such as caffeine. As many of the active components in the Coffea arabica are antioxidant compounds, upon administration, they scavenge free radicals, protect against oxidation of low-density lipoprotein (LDL), and inhibit cell damage due to reactive oxygen species (ROS). This inhibits oxidative stress and protects against DNA damage. The components in the extract also inhibit enzymes involved in inflammation, cell replication and DNA synthesis, and induce the expression of antioxidant enzymes. They also induce anti-inflammatory-mediated pathways, decrease nuclear factor KappaB (NF-kB) activation and decrease the expression of pro-inflammatory cytokines. This may inhibit growth and induce apoptosis of cancer cells. In addition, Coffea arabica extract upregulates the expression of anti-inflammatory adipokines (adipocytokines), such as adiponectin, and reduces the expression of pro-inflammatory adipokines, such as tumor necrosis factor-alpha (TNF-a), leptin and interleukin 6 (IL-6), thereby further preventing inflammation and inflammation-induced cancer. Adipokines are normally secreted by adipose tissue and play a key role in the regulation of glucose and fat metabolism, insulin sensitivity and inflammation. 